\title{
Prevalencia de lesiones de bajo y alto grado de cuello uterino en una ciudad colombiana
}

\author{
Luis Alfonso Mendoza T. 1,2, Marly Julieta Pedroza P. 2, Paul Hernando Micolta C. 2 , \\ Andrés Ramirez R. 2, Christian Ramiro Cáceres G. 2, Darling Viviam López S. 2, Anto- \\ nio José Nuñez G. 2, Maria Acuña P. 2 \\ 1 División de Epidemiología Hospitalaria, Fundación Hospital San José de Buga. ${ }^{2}$ Unidad Central del Valle, \\ Tuluá, Colombia.
}

\section{RESUMEN}

Objetivo: Determinar la prevalencia de las lesiones de bajo y alto grado de cuello uterino en una Institución Prestadora de Servicios de Salud de Tuluá, Colombia, entre los años 2008-2010. Métodos: Se realizó un estudio descriptivo retrospectivo en el que se incluyeron mujeres entre los 15 y 75 años, residentes en el Municipio de Tuluá. Se evaluó la normalidad de las variables cuantitativas, empleándose la mediana o el promedio con sus varianzas según su distribución, y frecuencias y proporciones para las variables categóricas. Resultados: Se incluyeron 3.539 mujeres. La mediana de la edad fue 38 años con rango intercuartil (RI: 29-46 años), la mediana del número de partos 1 (RI: 0-3). En relación a la seguridad social y uso de métodos anticonceptivos, la mayoría eran particulares y no utilizaban ningún método. El $87,5 \%$ de las citologías se reportaron como negativas, $8,2 \%$ lesión de bajo grado (LSIL), 2,6\% células escamosas atípicas de significado indeterminado (ASC-US), 0,9\% lesiones de alto grado (HSIL), 0,4\% células escamosas atípicas de significado indeterminado de alto grado ASC-H, 0,4\% células glandulares atípicas de significado incierto (ASGUS) y $0,2 \%$ carcinoma invasor. Conclusión: La prevalencia de las anormalidades citológicas fue baja en todos los grupos analizados, sin embargo, existe un mayor número de LSIL hacia la cuarta década de la vida y en aquellas que iniciaron su vida sexual después de los 40 años. De igual manera se encontró una relación inversa entre el número de partos, planificación familiar hormonal y aparición de lesiones pre neoplásicas.

\section{PALABRAS CLAVES: Citología cervicouterina, cáncer de cuello uterino, virus del papiloma humano}

\section{SUMMARY}

Objective: To determine the prevalence of lesions of low and high grade cervical in a Lender Institution Health Services, Tuluá, Colombia, 2008-2010. Methods: We performed a retrospective study which included women between 15 and 75 years living in Tuluá. We evaluated the normality of quantitative variables, using the median or average with their variances for quantitative variables according to their distribution and frequencies and proportions for categorical variables. Results: 3539 women were included. The median age was 38 years with interquartile range (IR: 29-46 years) and the median parity was 1 (IR: 0-3). In relation to social security and family planning birth control, most were private and did not use any method. The $87.5 \%$ of smears was reported as negative, low-grade squamous intraepitelial lesions (LSIL) $8.2 \%$, atypical squamous cells of undetermined significance (ASC-US) 2.6\%, high-grade squamous intraepitelial lesions (HSIL) $0.9 \%$, atypical squamous cells cannot rule out a high grade lesion (ASC-H) $0.0 \%$, atypical squamous glandular cells of undetermined significance (ASGUS) $0.4 \%$ and invasive carcinoma $0.2 \%$. Conclusion: The prevalence of cytological abnormalities was low in all groups studied, however, a greater number of LSIL to 
the fourth decades of life and those who began their sexual life after 40 years. Similarly, an inverse relationship was found between parity, hormonal family planning and pre-neoplastic lesions.

\section{KEY WORDS: Cervical cytology, cervical cancer, human papilloma virus}

\section{INTRODUCCIÓN}

El cáncer cervicouterino sigue siendo la primera causa de morbimortalidad en mujeres de países en vías de desarrollo. Los datos estadísticos mundiales indican que al año se producen unos 466.000 nuevos casos, $80 \%$ de ellos en países en vías de desarrollo, de los cuales mueren 274.000 mujeres, a pesar de ser una enfermedad prevenible (1). Para Colombia en el año 2002, se diagnosticaron 6.800 nuevos casos de cáncer de cuello uterino y se registraron 2.045 muertes por esta causa, siendo la primera causa de muerte por cáncer y la principal causa de muerte relacionada con la salud sexual y reproductiva (2).

Esta variación en la incidencia puede explicarse debido a los distintos métodos para su enfoque utilizados por los diferentes países y a diferentes factores entre los que se destacan: nivel de experiencia de los profesionales de la salud, baja cobertura de citología cervicouterina, limitada capacidad de consultorios para la toma de citología, laboratorios de lectura dispersos sin adecuado control de calidad, entre otros (1).

En las últimas décadas los programas de tamización citológica han reducido sustancialmente la incidencia y mortalidad del cáncer cervical en los países desarrollados, siendo aún la segunda causa de muerte en mujeres alrededor del mundo $(3,4)$. El Programa Nacional de Cáncer Cérvico Uterino de Chile logró reducir de manera significativa la tasa de mortalidad ajustada por este cáncer desde $14,3 / 100.000$ en 1990 a 8,5/100.000 mujeres en 2003. El Programa mostró una reducción anual de la tasa bruta de 0,268/100.000 mujeres y de la ajustada de $0,463 / 100.000$ mujeres, estimando para el 2010 una tasa bruta de mortalidad de 6,4/100.000 y para la ajustada por edad de 4,8/100.000, cifra menor que el objetivo propuesto por el Ministerio de Salud Pública de Chile (5). A pesar de que Colombia alcanzó una cobertura de citología superior al $70 \%$, tras más de quince años de introducir los programas de tamización, no se ha logrado disminuir significativamente la mortalidad por esta causa (6). Adicional a la falta de impacto, y como indicador de las deficiencias en los programas de tamización, no existen datos acerca de las lesiones de alto grado o cáncer cervical, lo cual es indispen- sable para organizar campañas de prevención y promoción, así como de diagnóstico, tratamiento y seguimiento oportuno para mejorar el pronóstico de esta patología (7). En consecuencia, la ausencia de información conduce a una deficiente planificación de programas y servicios, por subestimar los recursos necesarios y no identificar adecuadamente la población en riesgo de cáncer de cuello uterino (6).

El objetivo de esta investigación fue determinar la prevalencia de las lesiones de bajo y alto grado de cuello uterino en una Institución Prestadora de Servicios de Salud (IPS), representativa de la población de mujeres del municipio de Tuluá, Colombia.

\section{PACIENTES Y MÉTODOS}

Diseño de investigación y población de estudio: Se realizó un estudio descriptivo retrospectivo entre mujeres de 15 a 75 años que residen en el Municipio de Tuluá, Colombia, y que asistieron a la toma de citología a la IPS Unicáncer Capítulo Tuluá, entre los años 2008 y 2010. La población se limitó a este rango de edad debido a que las mujeres menores de 15 y mayores de 75 años no asisten con frecuencia a la toma de citología cervical, además que en anteriores investigaciones se han tomado estos rangos de edad, por lo que serían útiles para comparaciones, además de la baja incidencia de las patologías cervicouterinas en estas edades extremas. La selección de la entidad pública Unicáncer Capítulo Tuluá, se hizo por su alto índice de toma de citologías anuales en esta ciudad, a la calidad del procedimiento y sus resultados, además, por ser representativa de la mujer urbana, y que permitiría extrapolar los hallazgos.

Criterios de inclusión y exclusión: Mujeres en rangos de edad entre 15 y 75 años, residentes del Municipio de Tuluá. Se excluyeron embarazadas, histerectomizadas, conizadas previas antes del año 2008, y las con infección que pudiera alterar los resultados de la citología.

Variables analizadas: Edad, diagnóstico (lesiones de bajo o alto grado y presencia de cáncer de cuello uterino), paridad, tipo de seguros en salud, método de planificación familiar y sexarquia.

Tamaño de la muestra y muestreo. El tamaño de la muestra se obtuvo mediante el programa Epidat $₫$ 3.1. Para ello se tuvo en cuenta una población de 
76.655 mujeres Tulueñas de 15 a 75 años, con una proporción esperada de las patologías de $10 \%$, un error absoluto de $1 \%$, un nivel de confianza de $95 \%$ y efecto de diseño de 1\%. El tamaño de muestra así calculado fue de 3.342 mujeres. Sin embargo, se aumento el tamaño de la muestra en un $5 \%$ teniendo en cuenta que podrían haber datos faltantes. Es así, como mediante un muestreo probabilístico tipo aleatorio simple se tuvo un tamaño de 3.539 mujeres para el estudio.

Fuente de información. Como fuente de información se utilizó los registros de los resultados citológicos realizados entre los años 2008 al 2010 por Unicáncer Capítulo Tuluá. El método de recolección fue la extracción directa de los datos de dichos registros, previa capacitación y entrenamiento del personal.

Análisis estadístico. Los datos obtenidos se recogieron en una base de datos diseñada en el programa Microsoft Office Excel 2007. Después fueron llevados al paquete estadístico Stata ${ }^{2} 11.0$, donde se realizaron los análisis. Inicialmente se hizo un análisis exploratorio, evaluando la normalidad de la distribución de las variables cuantitativas mediante las pruebas de skewness, kurtosis y Shapiro-Francia. Las variables continuas se expresaron acordando su distribución y las variables categóricas se expresaron en frecuencias y proporciones. Algunas variables se agruparan en rangos para su análisis. Finalmente, las patologías fueron evaluadas por grupos de edad y otras variables como edad de inicio de la actividad sexual, método de planificación familiar y número de partos.

Consideraciones éticas. Para la realización de esta investigación, se contó con la aprobación del Comité de Ética e Investigaciones de la Unidad Central del Valle del Cauca y la aprobación de las directivas de Unicáncer Capítulo Tuluá. El estudio es catalogado sin riesgo y carece de conflicto de intereses.

\section{RESULTADOS}

Sobre la población en estudio de 3.539 mujeres, la mediana de la edad fue de 38 años (RI: 29-46 años), la mediana de edad de inicio de la actividad sexual de 17 años (Rl: 16-19 años) y la mediana del número de partos de 1 (Rl: $0-3$ ). El $71 \%$ de las mujeres tuvieron una edad entre los 16-44 años; el $74,8 \%$ inició la actividad sexual entre los 16 y 24 años y tenían menos de 3 partos, y el $49,6 \%$ no realizaba planificación familiar (Tabla I).

El $87,5 \%$ de las 3.539 citologías fueron informadas como negativas. El $8,2 \%$ fueron lesiones de bajo grado, 0,9\% de alto grado, 2,6\% ASC-US, $0,4 \%$ ASC-H, $0,4 \%$ ASGUS y $0,2 \%$ lesión compatible con cáncer invasivo (Tabla II).
Tabla I

\section{CARACTERÍSTICAS SOCIODEMOGRÁFICAS DE LA POBLACIÓN EN ESTUDIO (n: 3.539 MUJERES)}

\begin{tabular}{lrr}
\hline Variable & $\mathrm{n}$ & $\%$ \\
\hline Rango de edad (años) & & \\
$14-15$ & 7 & 0,2 \\
$16-34$ & 1382 & 39,1 \\
$35-44$ & 1127 & 31,9 \\
$45-64$ & 905 & 25,6 \\
$>65$ & 118 & 3,3 \\
Sexarquia (años) & & \\
$14-15$ & 739 & 20,9 \\
$16-24$ & 2648 & 74,8 \\
$25-34$ & 141 & 4,0 \\
$>35$ & 11 & 0,3 \\
Número de partos & & \\
0-2 & 2644 & 74,7 \\
3-5 & 771 & 21,8 \\
6-18 & 124 & 3,5 \\
Planificación familiar & & \\
Ninguno & 1755 & 49,6 \\
Dispositivo intrauterino & 113 & 3,2 \\
Vasectomía & 62 & 1,8 \\
Ligadura tubaria & 936 & 26,5 \\
Hormonal & 504 & 14,2 \\
Barrera & 121 & 3,4 \\
Otros & 48 & 1,4 \\
\hline
\end{tabular}

\section{Tabla II}

RESULTADOS DE LA CITOLOGÍA (n: 3.539 MUJERES)

\begin{tabular}{|c|c|c|}
\hline Citología & $\mathrm{n}$ & $\%$ \\
\hline Negativas & 3.095 & 87,5 \\
\hline $\begin{array}{l}\text { Lesión de bajo grado (NIC I y } \\
\text { cambios por VPH) }\end{array}$ & 290 & 8,2 \\
\hline $\begin{array}{l}\text { Lesión de alto grado (NIC II, NIC } \\
\text { III y Carcinoma in situ) }\end{array}$ & 30 & 0,9 \\
\hline Cáncer invasor & 6 & 0,2 \\
\hline ASC-US & 91 & 2,6 \\
\hline ASC-H & 14 & 0,4 \\
\hline ASGUS & 13 & 0,4 \\
\hline
\end{tabular}

ASC-US: Células escamosas atípicas de significado indeterminado. ASC-H: Células escamosas atípicas de significado indeterminado de alto grado. ASGUS: Células glandulares atípicas de significado incierto. 


\section{Tabla III}

ANORMALIDADES CITOLÓGICAS POR GRUPOS DE EDAD

\begin{tabular}{lcccrc}
\hline Citología & $\begin{array}{c}14-15 \mathrm{a} \\
\mathrm{n}(\%)\end{array}$ & $\begin{array}{c}16-34 \mathrm{a} \\
\mathrm{n}(\%)\end{array}$ & $\begin{array}{c}35-44 \mathrm{a} \\
\mathrm{n}(\%)\end{array}$ & $\begin{array}{c}45-64 \mathrm{a} \\
\mathrm{n}(\%)\end{array}$ & $\begin{array}{c}>65 \mathrm{a} \\
\mathrm{n}(\%)\end{array}$ \\
\hline Negativo & $7(100)$ & $1230(89)$ & $945(83,9)$ & $802(88,7)$ & $111(94)$ \\
Lesión de bajo grado & 0 & $99(7,2)$ & $125(11,1)$ & $64(7,1)$ & $2(1,7)$ \\
Lesión de alto grado & 0 & $12(0,9)$ & $10(0,9)$ & $6(0,7)$ & $2(1,7)$ \\
Cáncer invasor & 0 & 0 & $5(0,4)$ & $1(0,1)$ & 0 \\
ASC-US & 0 & $37(2,7)$ & $33(2,9)$ & $20(2,2)$ & $1(0,8)$ \\
ASC-H & 0 & $1(0,1)$ & $6(0,5)$ & $5(0,6)$ & $1(0,8)$ \\
ASGUS & 0 & $3(0,2)$ & $3(0,3)$ & $6(0,7)$ & $1(0,8)$
\end{tabular}

ASC-US: Células escamosas atípicas de significado indeterminado. ASC-H: Células escamosas atípicas de significado indeterminado de alto grado. ASGUS: Células glandulares atípicas de significado incierto.

En el grupo de 14-15 años todas las citología fueron informadas como negativas, mientras en el grupo de 16-34 años el 7,2\% ( $n=99)$ presentaron lesiones de bajo grado, siendo el grupo de 35-44 años el más afectado por este tipo de lesiones con $11,1 \%(n=125)$. El grupo de edad con mayor número de mujeres afectadas por lesiones de alto grado, cáncer invasivo, ASC-US y ASC-H fue el de 3544 años, mientras las lesiones ASGUS afectó en mayor proporción a las mujeres entre 45-64 años (Tabla III). El carcinoma invasor obtuvo porcentajes entre $0,4 \%(n=5)$ y $0,1 \%(n=1)$ para los grupos entre 35-44 años y 45-64 años respectivamente, estando ausente en los demás grupos de edad (Tabla III).

En relación al inicio de la actividad sexual, el $64 \%$ de las lesiones cervicouterinas se encontraron en mujeres que iniciaron la actividad sexual entre los 15 y 19 años, seguido por el grupo de 20 y más años $(26,4 \%)$.

El $47,7 \%$ de las lesiones se presentaron en mujeres que no utilizaban ningún método de planificación familiar, seguida de la ligadura de trompas $(30,4 \%)$, método hormonal $(10,4 \%)$, mientras que la menor frecuencia de lesiones se presentó en las usuarias métodos de barrera $(3,6 \%)$ (Tabla IV). El mayor porcentaje de lesiones de bajo grado ocurrieron en mujeres que no utilizaban ningún método de planificación familiar $(3,9 \%)$, seguido por aquellas que tenían ligadura de trompas $(2,7 \%)$ y por aquellas que planificaban con método hormonal $(0,6 \%)$. Las lesiones de alto grado ocurrieron principalmente en mujeres igualmente sin planificación familiar $(0,4 \%)$, seguido por aquellas que tenían ligadura de trompas $(0,1 \%)$ o planificaban con método hormonal $(0,1 \%)$ (Tabla V).

En cuanto a la paridad se observó que las mujeres con 0 partos y 1 a 2 partos, fueron las que mayor porcentaje de lesiones tuvieron $(37,2 \%$ cada uno), siendo del $5 \%$ en las mujeres con 5 y más partos (Tabla IV). Según el tipo de lesión cervicouterina, la distribución estuvo a favor de las mujeres que tenían entre 0-3 partos: lesiones de bajo grado 0 partos (75,2\%); lesiones de alto grado 0 a 3 partos (90\%); cáncer invasor 0 partos (50\%); ASC-US 0 a 3 partos $(92,3 \%)$; ASC-H 0 a 2 partos $(78,6 \%$ ) y ASGUS 0 a 2 partos $(83,3 \%)$.

La mayor parte de las mujeres acudieron a la toma de citología particularmente, mientras que sólo el $2,6 \%$ lo hizo a través del plan obligatorio de salud.

\section{DISCUSIÓN}

La prevalencia de alteraciones citológicas sobre un universo de 3.539 mujeres fue de $12,5 \%$, de ellas el $8,2 \%$ correspondió a lesiones de bajo grado y el $0,9 \%$ a lesiones de alto grado. Estos resultados son inferiores a los informados en Colombia por González y cols (6), quienes hallaron un 30,6\% de alteraciones en la citología cervicouterina en un estudio sobre 4.957 mujeres en Bogotá, pero más elevada que lo informado por otros autores para quienes la prevalencia estuvo entre 0,2-9\% (8-11). En otras investigaciones, la prevalencia de anormalidades citológicas varía según el tipo de población en estudio y los factores de riesgos inherentes a la misma. En una muestra de 31 pacientes VIH positivas asintomáticas desde el punto de vista ginecológico, se reportó una alta prevalencia (18/31) de lesiones intraepiteliales escamosas de alto y bajo grado (12). 
Tabla IV

ANORMALIDADES CITOLÓGICAS SEGÚN EDAD DE INICIO DE LA ACTIVIDAD SEXUAL, MÉTODOS DE PLANIFICACIÓN FAMILIAR Y NÚMERO DE PARTOS

\begin{tabular}{lccr}
\hline Grupo de edad de inicio de actividad sexual & $\mathrm{n}$ & Lesiones & $\%$ \\
9-14 años & 314 & 24 & 5,4 \\
$15-19$ años & 2323 & 284 & 64,0 \\
20 y más años & 870 & 117 & 26,4 \\
Sin datos & 32 & 19 & 4,3 \\
Total & 3539 & 444 & 100,0 \\
Método de planificación & $\mathrm{n}$ & Lesiones & $\%$ \\
$\quad$ Ninguno & 1755 & 212 & 47,7 \\
Dispositivo intrauterino & 113 & 20 & 4,5 \\
Vasectomía de pareja & 62 & 12 & 2,7 \\
Ligadura tubaria & 936 & 135 & 30,4 \\
Hormonal & 504 & 46 & 10,4 \\
Barrera & 121 & 16 & 3,6 \\
Otro & 48 & 3 & 0,7 \\
Total & 3539 & 444 & 100,0 \\
Paridad & $\mathrm{n}$ & Lesiones & $\%$ \\
0 & 1040 & 165 & 37,2 \\
1 a 2 & 1604 & 1650 & 37,2 \\
3 a 4 & 690 & 92 & 20,7 \\
$\geq 5$ & 205 & 22 & 5,0 \\
Total & 1040 & 165 & 100,0 \\
\hline
\end{tabular}

Tabla V

DISTRIBUCIÓN DE LAS ANORMALIDADES CITOLÓGICAS SEGÚN MÉTODO DE PLANIFICACIÓN FAMILIAR

\begin{tabular}{lrrrrrr}
\hline Método de planificación & $\begin{array}{c}\text { LBG } \\
(\%)\end{array}$ & $\begin{array}{c}\text { LAG } \\
(\%)\end{array}$ & $\begin{array}{c}\text { Ca invasor } \\
(\%)\end{array}$ & $\begin{array}{c}\text { ASC-US } \\
(\%)\end{array}$ & $\begin{array}{c}\text { ASC-H } \\
(\%)\end{array}$ & $\begin{array}{c}\text { ASGUS } \\
(\%)\end{array}$ \\
\hline Ninguno & $138(3,9)$ & $15(0,4)$ & $2(0,1)$ & $46(1,3)$ & $5(0,1)$ & $6(0,2)$ \\
Dispositivo intrauterino & $14(0,4)$ & $3(0,1)$ & $0(0,0)$ & $2(0,1)$ & $0(0,0)$ & $1(0,0)$ \\
Vasectomía de pareja & $8(0,2)$ & $1(0,0)$ & $0(0,0)$ & $3(0,1)$ & $0(0,0)$ & $0(0,0)$ \\
Ligadura de trompas & $94(2,7)$ & $5(0,1)$ & $2(0,1)$ & $26(0,7)$ & $5(0,1)$ & $3(0,1)$ \\
Hormonal & $23(0,6)$ & $4(0,1)$ & $2(0,1)$ & $12(0,3)$ & $2(0,1)$ & $3(0,1)$ \\
Barrera & $11(0,3)$ & $1(0,0)$ & $0(0,0)$ & $2(0,1)$ & $2(0,1)$ & $0(0,0)$ \\
Otro & $2(0,1)$ & $1(0,0)$ & $0(0,0)$ & $0(0,0)$ & $0(0,0)$ & $0(0,0)$ \\
Total & $290(8,2)$ & $30(0,9)$ & $6(0,2)$ & $91(2,6)$ & $14(0,4)$ & $13(0,4)$
\end{tabular}

ASC-US: Células escamosas atípicas de significado indeterminado. ASC-H: Células escamosas de significado indeterminado de alto grado. ASGUS: Células glandulares atípicas de significado incierto. LBG: Lesión de bajo grado. LAG: Lesión de alto grado. 
En países de América Latina, los datos de prevalencia de alteraciones citológicas son muy variables y se toman grupos poblacionales restringidos, tales como México con 3,4\% (13), Venezuela 13,2\% (14) y Ecuador 9,8\% (15), la gran mayoría de estas alteraciones corresponde a ASC-US, mientras que en el actual estudio la mayor prevalencia es de NIC I con $8,2 \%$ y en relación con ASC-US de $2,6 \%$, en contraste también al estudio realizado en Bogotá por González y cols (6), quienes reportan ASC-US del $24,7 \%$. Kasamatsu y cols (16), informan que a pesar de que en Paraguay la tamización con citología cervicovaginal no supera el $10 \%$, hubo un incremento en resultados alterados entre 2001 y 2006 de $0,5 \%$ a $1,1 \%$. Schmolling y cols (17), en un estudio con 5.712 citologías realizado en España, encontraron un total 308 (5,4\%) anormalidades epiteliales cervicales. Por su parte, en Estados Unidos de Norte América esta alteración es hallada en diferentes estudios entre 7 y $23 \%(18-20)$.

La mayor parte de las alteraciones citológicas vaginales estuvo concentrada en el grupo de lesiones de bajo grado (NIC I y cambios por VPH) con un $9,2 \%$, seguido por ASC-US 2,6\%, lesiones de alto grado (NIC II, NIC III y carcinoma in situ) 0,9\%, ASCH 0,4\%, ASGUS 0,4\% y cáncer invasor 0,2\%. Nuestros resultados contrastan con los de Bravo y cols (21), en un estudio realizado en Popayán, Colombia, con 416 mujeres, los cuales hallaron un 7,9\% de alteraciones en la citología cervicouterina, entre las cuales la mayor proporción fueron ASC-US 5,5\%, lesiones de bajo grado $1,9 \%$ y atipia endocervical $0,5 \%$ y con los de Schmolling y cols (17), quienes entre las 308 citologías alteradas, hallaron que más de la mitad eran anomalías de significado indeterminado: ASC-US 101 (32,8\%), y ASGUS 60 (19,5\%), seguido por LBG 90 (1,57\%), LAG 54 (0,94\%), carcinoma escamoso $2(0,035 \%)$, y adenocarcinoma $1(0,01 \%)$. González y cols (6), hallaron entre sus 1.530 citología alteradas que lesiones ASC-US representaron la mayor proporción $24,7 \%$, seguido de LBG 4,3\%, de LAG 1,2\%, ASGUS 0,26\% y citología sospechosa de cáncer de 0,1\% (6).

En cuanto a la edad, se encontró que las anormalidades citológicas están ausentes en menores de 16 años, muy escasas en las mayores de 64 años, siendo más frecuentes en los grupos de 3544 años, 16-34 años y 45-64 años, datos que han sido informados previamente $(4,22,23)$. En el presente estudio, las lesiones preneoplásicas no se relacionaron con la multiparidad, contrario a la literatura, que describe displasia y carcinoma in situ 5 veces más frecuente en multípara que en primíparas, frecuencia que aumenta con el número de hijos hasta el punto de ser el doble en la multípara que en la primípara (24). En algunos estudios se cree que esta relación corresponde al efecto de factores hormonales asociados al embarazo o al traumatismo cervical del parto (22).

Las mujeres sin planificación familiar fueron las que con mayor frecuencia presentaron lesiones (de bajo grado 3,9\%, alto grado 0,4\%, cáncer invasor $0,1 \%$, ASC-US $1,3 \%$, ASC-H 0,1\% y ASGUS $0,1 \%$ ), seguido por aquellas que tenían ligadura de trompas (de bajo grado 2,7\%, alto grado 0,1\%, cáncer invasor 0,1\%, ASC-US 0,7\%, ASC-H 0,1\% y ASGUS 0,1\%), mientras que aquellas que planificaban con método hormonal ocuparon el tercer lugar (de bajo grado 0,6\%, alto grado 0,1\%, cáncer invasor $0,1 \%$, ASC-US 0,3\%, ASC-H 0,1\% y ASGUS $0,1 \%$ ). En la literatura se sugiere que el uso a largo plazo (5 o más años) de los anticonceptivos orales podía aumentar hasta 4 veces el riesgo de cáncer cervicouterino en las mujeres infectadas con VPH, aunque el riesgo tiende a desaparecer en los 5 años siguientes a la interrupción del consumo $(22,25)$. Para otros autores, la frecuencia de citologías positivas en mujeres con tratamiento hormonal es inferior a la de mujeres sin este tratamiento $(p=$ $0,029)$, mientras la frecuencia de lesiones precancerosas en mujeres con tratamiento hormonal también es inferior a la de mujeres sin este tratamiento $(p=0,012)$ (17). En nuestro trabajo las mujeres que no usan métodos anticonceptivos quizás descuidan sus controles ginecológicos, lo que hace que se expongan más a infección por VPH. Si revisamos los 320 casos de lesiones de bajo y alto grado, encontramos que el 3,8\% (n: 12) ocurrieron en mujeres que usaron métodos de barrera, mientras que las que no planificaron presentaron el 47,8\% (n: 153) de los casos y las que usaron otro método de planificación diferente a los de barrera presentaron el $48,4 \%$ (n: 155). Podemos inferior entonces, que la menor frecuencia de lesiones en usuarias de métodos de barrera, se deba a que tienen menos posibilidades de contacto con VPH.

Se encontró una mayor relación con lesiones pre-neoplásicas en pacientes con inicios tardíos de las relaciones sexuales (>35 años) con un 18\%, sin embargo, son pocos los casos. Lo que se espera, es que las mujeres con sexarquia antes de los 20 años, tengan 2 a 3 veces mayor riesgo de cáncer cervicouterino (26), pero esta asociación no se encontró en el presente estudio. Bravo y cols (21), encontraron que el $34 \%$ de las mujeres con lesiones cervicovaginales habían iniciado la actividad sexual antes de los 16 años, el 57\% entre los 16 y 19 años y el $9 \%$ a los 20 y más años. Por otro lado, se debe señalar que la mayoría de las usuarias de la IPS donde se realizó el estudio acuden como pacien- 
tes particulares, independiente que si tengan o no seguridad social en salud, con la salvedad, que en nuestro país, la citología cervicouterina está incluida dentro de los planes obligatorios de salud.

\section{CONCLUSIONES}

En el estudio realizado hallamos una prevalencia de lesiones de bajo grado de $8,2 \%$ y de alto grado de $0,9 \%$, observando una baja frecuencia de cáncer invasor $(0,2 \%)$. Nuestros hallazgos muestran que la prevalencia de las lesiones cervicouterinas son inferiores a las reportadas por otros autores en nuestro país $(30,6 \%)$, y en el rango de las informadas en la literatura latinoamericana y mundial $(3,4-23 \%)$. La mayor frecuencia de lesiones de bajo y alto grado del cuello cervicouterino afectó a la población entre 16-64 años, y el cáncer invasor a las mujeres entre 35-64 años. La mayor prevalencia de lesiones de cuello cervicouterino afectó a las mujeres que no realizaban planificación familiar, mientras que las usuarias de métodos de barrera presentaron la prevalencia más baja $(3,8 \%)$, sugiriendo su efecto protector. Se destaca la baja frecuencia de cáncer invasor, lo cual puede evidenciar que en la población se realiza una buena tamización, haciendo posible la detección de lesiones intraepiteliales, disminuyendo la morbimortalidad por cáncer cervicouterino.

AGRADECIMIENTOS: Los integrantes del grupo de investigación agradecen a los ejecutivos de Unicáncer Capítulo Tuluá, Colombia y a los dirigentes de la Unidad Central del Valle (UCEVA), Colombia, por sus desinteresados esfuerzos en brindar la colaboración necesaria para la realización del presente trabajo.

\section{REFERENCIAS}

1. Liga Colombiana Contra el Cáncer, 2009. Campaña de Prevención de Cáncer de Cuello Uterino [Internet]. Disponible en: http://www.ligacancercolombia.org/. Acceso el 03 de diciembre de 2011.

2. Cendales R, Piñeros $M$, Wiesner $C$, Murillo R, Tovar S. Cobertura de la citología de cuello uterino y factores relacionados en Colombia. Rev Colomb Cancerol 2008;12:119-25.

3. Vesco K, Whitlock E, Eder M, Burda B, Senger C, Lutz K. Risk factors and other epidemiologic considerations for cervical cancer screening: a narrative review for the U.S. Preventive Services Task Force. Ann Intern Med 2011;155:698-705.

4. Kalliala I, Dyba T, Nieminen P. Mortality in a longterm follow-up after treatment of CIN. Int $\mathrm{J}$ Cancer 2010;126:224-31.

5. Donoso E, Cuello M, Villarroel del P L. Reducción de la mortalidad por cáncer cérvico uterino en Chile, 19902003. Rev Chil Obstet Ginecol 2006;71:307-312.
6. González M, Murillo R, Osorio E, Gamboa O, Ardila J y Grupo de investigación de tamización en cáncer de cuello uterino. Prevalencia de anormalidades citológicas e histológicas de cuello uterino en un grupo de mujeres en Bogotá, Colombia. Rev Colomb Cancerol 2010;14:22-8.

7. Ries G, Melbert D, Krapcho M. SEER Cancer Statistics. [publicación periódica en línea] 2005 [citado 2011 Dic. 3]; [10 pantallas]. Disponible en: http://seer.cancer.gov/csr/1975_2005/,\%20based\%20on\%20November\%202007\%20SEERdata\%20submission,\%20 posted $\% 20$ to $\% 20$ the $\% 20$ SEER\%20web\%20site,\%20 2008. Acceso el 03 de noviembre de 2011.

8. Olazábal JC, Montero J, Pastor F, Alario MJ, García R, García JF. Diez años de citologías de cérvix uterino en un centro de salud. Aten Primaria 1997;20:293-8.

9. Valero F, Nebot MJ, Fenollosa B, Covira A, Rius J. Correlación entre el diagnóstico colposcópico y citohistológico en 285 casos de CIN. Prog Obstet Ginecol 1989;32:40-2. 29.

10. Gimeno A, Jiménez R, Camps del Bosque JR. Cáncer de cuello uterino en Extremadura. Rev San Hig Pub 1993;67:217-25.

11. Borrego JA, Santaella M, Márquez AJ, Martínez Cabral C, Arjona JE. Incidencia de patología cervical inflamatoria y preneoplásica en una consulta de ETS. Prog Obstet Gineco 1988;31:635-9.

12. Rodríguez G, Rivero I, Milan J, Cappuccio P, Lowinger M, Mirazo I, et al. Prevalencia de lesiones preneoplásicas y condiloma de cuello uterino en una muestra de pacientes VIH positivas de Uruguay. Rev Med Uruguay 1996;12:135-8.

13. Coronel P. Estudio exploratorio de las lesiones premalignas en el reporte citológico del cuello uterino. Rev Méd Universidad Veracruzana 2003;3(1). Disponible en: http://www.uv.mx/rm/num_anteriores/revmedica\%20vol3_num1/vol3_num1/articulos/estudio_ expl_lesiones_prem.html. Acceso el 16 de noviembre de 2011.

14. Universidad Centroccidental "Lisandro Alvarado", Decanato de Medicina. Frecuencia de alteraciones citológicas de cuello útero y los factores de riesgo asociados en las pacientes que acuden al Ambulatorio Urbano tipo II "Dr. Agustín Zubillaga", Barquisimeto, Estado Lara, Enero-Mayo 2005. Disponible en: http:// bibmed.ucla.edu.ve/cgi-win/be_alex.exe.BM-UCLA. Acceso el 16 de junio de 2011.

15. Sánchez H, Ortiz E, Vásquez M, Avila L, Campoverde N. Prevalencia de alteraciones citológicas por Pap y factores de riesgo para cáncer de cuello uterino en mujeres de 35 a 64 años. Oncología 2005;15:153-7.

16. Kasamatsu E, Páez MI. Cáncer de cuello uterino y virus del papiloma humano en Paraguay. Perspectivas para la prevención primaria. Mem Inst Investig Cienc Salud 2006;4:58-63. Disponible en: http://www.iics.una.py/n/ pdf/revista/34.pdf. Acceso el 16 de junio de 2011.

17. Schmolling Y, Barquín JJ, Zapata A, Merino R, Rodríguez $\mathrm{B}$, León $\mathrm{E}$. Anomalías citológicas de cérvix y lesiones precancerosas subsecuentes en un área sanitaria. Aten Primaria 2002;29:223-9.

18. Simsir A, Brooks S, Cochran L, Bourquin P, loffe OB. 
Cervicovaginal smear abnormalities in sexually active adolescents. Implications for management. Acta Cytol 2002;46:271-6.

19. Kulasingam SL, Hughes JP, Kiviat NB, Mao C, Weiss NS, Kuypers JM, et al. Evaluation of human papillomavirus testing in primary screening for cervical abnormalities: comparison of sensitivity, specificity, and frequency of referral. JAMA 2002;288:1749-57.

20. Trimble C, Richards L, Wilgus-Wegweiser B, Plowden $K$, Rosenthal D, Klassen A. Effectiveness of screening for cervical cancer in an inpatient hospital setting. Obstet Gynecol 2004;103:310-6.

21. Bravo M, Erazo J, Álvarez A, Casas M, Ortiz O y Álvarez J. Prevalencia de anormalidades en la citología cervical en tres grupos poblacionales de mujeres de Popayán, Colombia 2003-2005. Rev Colomb Obstet Ginecol 2008;59:190-8.

22. ACCP Recs 2007 Factsheet Spanish.pdf [Internet]. Hallado en: http://www.alliance-cxca.org/espanol/
ACCP_recs_2007_factsheet_Spanish.pdf. Acceso el 03 de diciembre de 2011.

23. Castellsagué $X$, Díaz $M$, Vaccarella $S$, de Sanjosé $S$, Muñoz N, Herrero R, et al. Intrauterine device use, cervical infection with human papillomavirus, and risk of cervical cancer: a pooled analysis of 26 epidemiological studies. Lancet Oncol 2011;12:1023-31.

24. Parsons L, Sommers SC. Cancer of the Cervix. In: Gynecology. Second edition. 1978:1300-1420. Parson L, Sommers SC (eds).

25. Diestro M, Serrano F, Gómez N. Cáncer de cuello uterino. Estado actual de las vacunas frente al virus del papiloma humano (VPH). Oncología 2007;30:42-59.

26. Márquez A, González J, Chávez F, Almirall A, Valdés R. Factores de Riesgo del Cáncer de Cérvix Uterino. Ginecología. [publicación periódica en línea] 2008. Ago [Citado 2011 Dic. 3]: [9 pantallas]. Hallado en: http://bvs.sld.cu/revistas/gin/vol34_2_08/gin07208. htm. 